\title{
Reconfiguración del trapecio rectángulo en una malla cuadriculada: una investigación con estudiantes peruanos de segundo grado de educación secundaria
}

\section{Reconfiguration of the rectangle trapezoid in a square grid: an investigation with Peruvian second graders of secondary education}

\author{
Isela Patricia Borja Rueda ${ }^{1}$ \\ iselaborja@yahoo.com \\ Verónica Neira Fernández ${ }^{2}$ \\ vneira@pucp.pe
}

\begin{abstract}
Resumen
El presente artículo es parte de la investigación de Borja (2015) cuyo objetivo es presentar una investigación con estudiantes peruanos de Educación Secundaria (12 a 15 años), acerca de la reconfiguración del trapecio rectángulo que está en una malla cuadriculada para hallar su medida de área. El trabajo se basa en aspectos de la Teoría de Registros de Representación Semiótica y de la metodología de la Ingeniería Didáctica. En la cual, se observa que los estudiantes realizan la descomposición heterogénea del trapecio rectángulo para transformarlo en una nueva figura geométrica que permita el conteo de los cuadrados completados en la malla cuadriculada, considerados como unidad de área, para determinar su medida de área. Es decir, realizan la operación de reconfiguración.
\end{abstract}

Palabras-clave: Reconfiguración; Rectangle trapezoid; Medida de área.

\begin{abstract}
This article is part of research Borja (2015) which aims to present research with Peruvian secondary school students (12-13 years), about the reconfiguration of the rectangle trapezoid is a grid mesh find it measure area. The work is based on aspects of Theory of Semiotics Representation Registers and Didactic Engineering methodology. In which it is observed that students take the heterogeneous decomposition of the isosceles trapezoid to transform it into a rectangle, that means reconfiguration and count the squares completed in the grid, considered unit area to determine its extent of area. Then, the perceptual support the grid mesh influences the reconfiguration.
\end{abstract}

Keywords: Reconfiguration; Geometric figures; Measure of area.

\section{Introducción}

Según la investigación de Borja (2015) los estudiantes peruanos del segundo grado de Educación Secundaria de una institución educativa pública, con edades comprendidas entre los 12 y 15 años de edad, para hallar la medida del área del trapecio utilizan la fórmula. La cual es muchas veces memorizada y utilizada de manera mecánica por los estudiantes. Por ello, se realizó una investigación cualitativa con dichos estudiantes para determinar la medida del área del trapecio a través de la operación de la reconfiguración. En base a aspectos de la Teoría de Registros de Representación Semiótica de Duval (2004) y de la Ingeniería Didáctica

\footnotetext{
${ }^{1}$ Institución Educativa Básica Regular Andahuasi - IREM-PUCP - a19999366@pucp.pe

${ }^{2}$ Pontificia Universidad Católica del Perú-IREM-PUCP- vneira@pucp.pe
} 
de Artigue (1995) como metodología de la investigación cualitativa. Sin embargo, para el artículo consideramos el análisis a priori y a posteriori de un trapecio rectángulo de la actividad 1: Trabajemos con la malla cuadriculada de la investigación de Borja (2015), porque esta figura geométrica está presente en su texto de Matemática 2 Secundaria (Perú, 2012). Definido el trapecio rectángulo como un cuadrilátero en el que uno de sus lados es perpendicular a las bases (Alva, 2015). Asimismo, Douady \& Perrín-Glorian (1987), mencionan que el uso prematuro de fórmulas para determinar la medida del área ha propiciado en los estudiantes que presenten inconvenientes al respecto. Por ello, la importancia del presente artículo que nos muestra una manera diferente de determinar la medida del área como es a través del uso de la operación de reconfiguración sin recurrir a la fórmula del área del trapecio.

\section{Aspectos de la Teoría de Registros de Representación Semiótica}

La Teoría de Registros de Representación Semiótica es propuesta en el año 1995. Duval (2004) la presenta de la siguiente manera, se debe requerir para aprender matemáticas de las representaciones porque los objetos matemáticos no son reales a diferencia de otras ciencias en que los objetos de estudio son reales como en el caso de la Biología. Estas son producidas por el sujeto y son expresadas, es decir, las representaciones son semióticas. Asimismo, el investigador señala que cuando estas representaciones semióticas cuentan con tres actividades cognitivas a saber: la formación, el tratamiento y la conversión, estas representaciones son considerados registros.

En tanto, Duval (2001), expresa que existen dos grandes tipos de transformaciones en los registros de representación semiótica que son: el tratamiento y la conversión. El primero es una transformación que se da dentro de un mismo registro, por ejemplo, la reconfiguración en el registro figural, al cual nos referiremos en el presente artículo.

También indica que son cuatro los registros de representación semiótica que movilizan las matemáticas: el registro de lengua natural, el registro algebraico, el registro figural y el registro gráfico. Pero, en este artículo nos referiremos al registro figural, que según Duval (1994) hay cuatro maneras de aprehender este registro en geometría por el estudiante: la aprehensión perceptiva, la aprehensión discursiva, la aprehensión secuencial y la aprehensión operatoria que es cuando el estudiante realiza modificaciones en la figura como la mereológica, que consiste en dividir o fraccionar en varias sub-figuras a la figura inicial para reagruparlas a través de la operación de reconfiguración.

Cabe señalar que la reconfiguración "es una operación que consiste en reorganizar una o 
varias sub-figuras diferentes de una figura dada en otra figura" (Duval, 2004, p. 165). Además, según el investigador esta operación no se presenta de manera espontánea y evidente, ya que pueden existir factores de soporte como la cuadrícula de fondo en la malla cuadriculada que permitirá a los estudiantes tener un soporte perceptivo, para realizar la operación de reconfiguración. De tal manera que, "permite iniciar, de inmediato, tratamientos como, la medida de área" (Duval, 1988, p. 64, traducción nuestra). Ya que se obtiene una nueva figura de contorno diferente a la figura inicial, pero que mantienen la misma medida de área.

Asimismo, para obtener estas sub-figuras se tiene que descomponer la figura inicial, cuyos tipos de descomposición de acuerdo a Duval (2005), son los siguientes: la descomposición estrictamente homogénea, descomposición homogénea y la descomposición heterogénea. Pero, en este trabajo nos referiremos a la descomposición heterogénea que se da en la figura cuando obtenemos unidades figurales de formas diferentes entre ellas (Figura 1).

Figura 1: Descomposición heterogénea

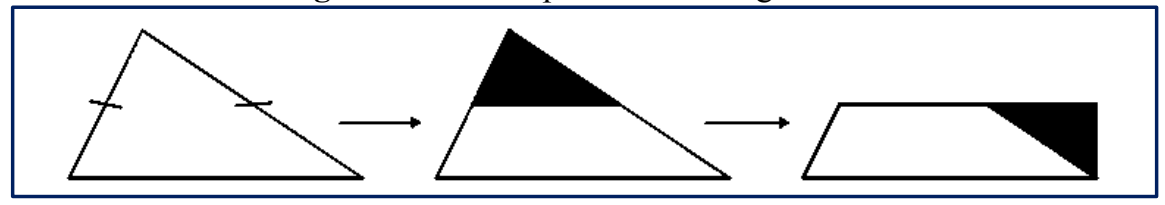

Fuente: Duval (2005, p. 22)

Según Duval (2004), las unidades figurales vienen hacer el cruce de los valores de la variable visual cualitativa con la variable de dimensión, que para este caso del trapecio isósceles el contorno cerrado de su superficie es una variable cualitativa y el área es una variable de dimensión dos.

\section{La investigación}

Borja (2015), utiliza aspectos de la Ingeniería Didáctica de Artigue (1995) como método de investigación. Para realizar la secuencia de tres actividades: Trabajemos con la malla cuadriculada, Trabajemos con el Geogebra y Hallemos la medida del área, esta última como actividad de cierre. Señalamos que las actividades desarrolladas de manera individual por diez estudiantes del segundo grado de Educación Secundaria de la Institución Educativa Básica Regular “Andahuasi” acompañados por la investigadora.

A priori lo que se espera es que los estudiantes realicen en el desarrollo de las actividades y en el análisis a posteriori, lo realizado por los estudiantes en dichas actividades. En tanto, el contraste del análisis a priori con el análisis a posteriori brinda la validación interna de la investigación. Presentamos el análisis a posteriori de tres estudiantes Melissa, Viviana y 
Alcides en relación a la medida del área del trapecio rectángulo que es una de las seis figuras geométricas de la actividad 1. También, las dos primeras respuestas de dicha actividad (Figura 2). Para lo cual, los estudiantes emplean los siguientes recursos: Ficha de la actividad 1, lápiz 2B y borrador.

Figura 2: Actividad 1: Trabajemos con la malla cuadriculada

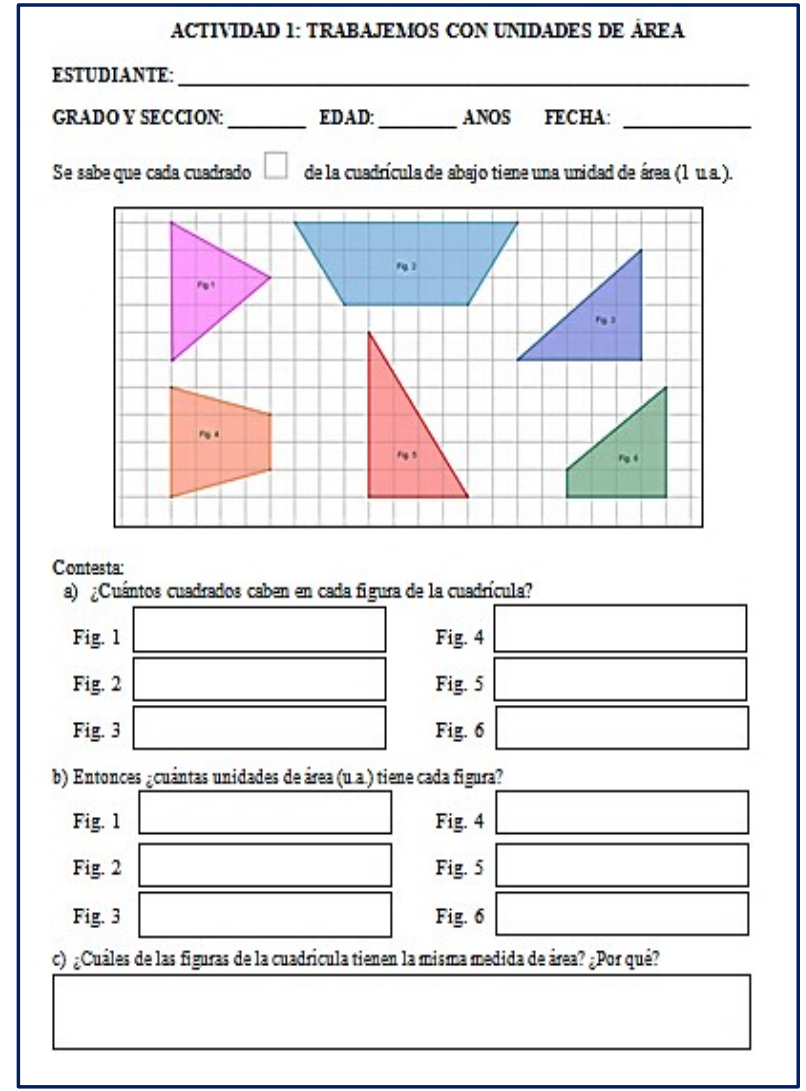

Fuente: Borja (2015, p. 49)

\section{Desarrollo de la actividad}

Para este artículo, de la investigación de Borja (2015) consideramos el análisis del trapecio rectángulo identificado con Fig. 6 como los ítems a) y b) de la Actividad 1: Trabajemos con la malla cuadriculada. Luego, con respecto al análisis a priori Borja (2015) afirma,

(...) esperamos que los estudiantes realicen dos trazos en el trapecio rectángulo para obtener un triángulo pequeño, luego lo trasladen según indica la flecha pequeña anaranjada y formen un rectángulo pequeño. Después, por aprehensión perceptiva identifiquen que en la parte superior del rectángulo pequeño hay un triángulo rectángulo que se puede trasladar para formar un rectángulo con ocho cuadrados de unidad de área, según indica la flecha verde. Por último, trasladarán el rectángulo pequeño hacia el lado derecho del rectángulo de ocho cuadrados de unidad de área, para tener un solo rectángulo de diez cuadrados de unidad de área, según indica la flecha roja que se observa en la reconfiguración inicial (pp. 52-53). (Ver Figura 3). 
Figura 3: Posible reconfiguración del trapecio isósceles

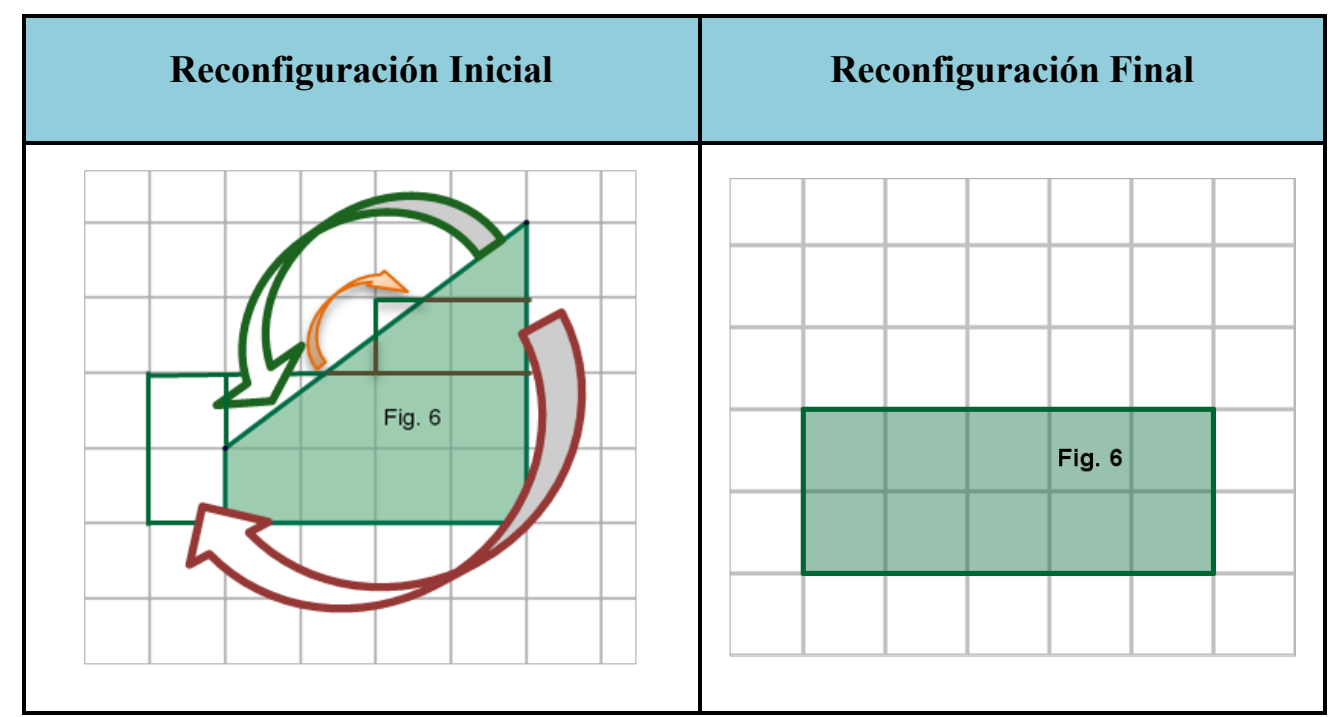

Fuente: Borja (2015, p. 51)

De esta manera esperamos que los estudiantes obtengan al realizar la modificación mereológica en el trapecio rectángulo (Fig. 6) cuatro sub-figuras que son las siguientes: dos triángulos y dos pentágonos. Luego, reagrupen estas sub-figuras, es decir, realicen la operación de reconfiguración y obtengan un rectángulo para a través de la estrategia del conteo determinen la medida de área. También, que contesten en la ficha de la actividad 1 el ítem a) ¿Cuántos cuadrados caben en cada figura de la cuadrícula? como el ítem b) Entonces ¿cuántas unidades de área (u.a.) tiene cada figura? y en relación al trapecio rectángulo las respuestas esperadas es 10 cuadrados y 10 u.a., respectivamente.

Es así que, en el análisis a posteriori la estudiante Melissa a diferencia de lo previsto en el a priori, al realizar la modificación mereológica en el trapecio rectángulo obtiene dos triángulos, un cuadrado y dos pentágonos, es decir, cinco sub-figuras, según las aspas y trazos hechos a lápiz por la estudiante en la Fig. 6. Pensamos que su aprehensión perceptiva fue influenciada por la cuadrícula de la malla para obtener estas sub-figuras. Luego, realizó la modificación posicional al trasladar dos triángulos (grande y pequeño), un cuadrado, un pentágono hacia el exterior de la figura inicial para formar un rectángulo. Luego, al reagrupar estas sub-figuras, es decir, la operación de reconfiguración obtiene un rectángulo como se esperaba. Es así, que completa los cuadrados de la figura inicial considerados como unidades de área. (Ver Figura 4). 
Figura 4: Descomposición heterogénea del trapecio rectángulo

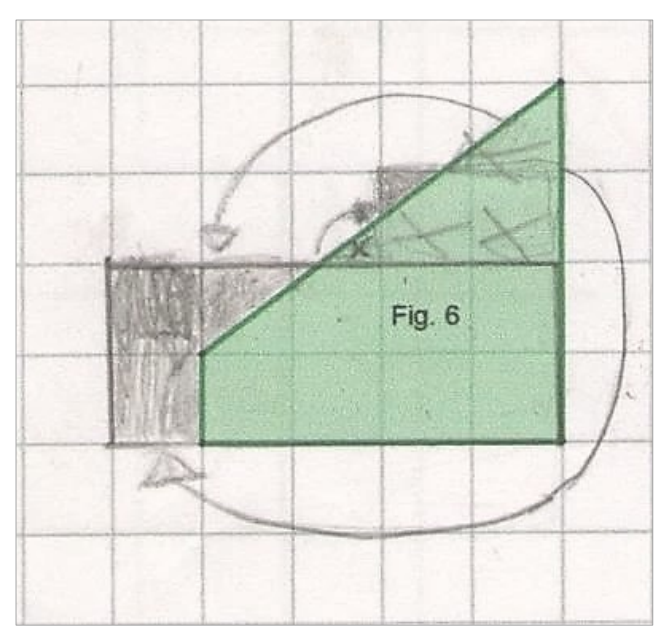

Fuente: Adaptado de Borja (2015, p. 54)

Borja (2015), indica que la estudiante Melissa responde a los ítems a) y b) de la actividad 1: Trabajemos con la malla cuadriculada según lo previsto en el a priori, al realizar la estrategia del conteo (ver Tabla 1).

Tabla 1: Respuestas de Melissa con respecto al trapecio rectángulo de la actividad 1

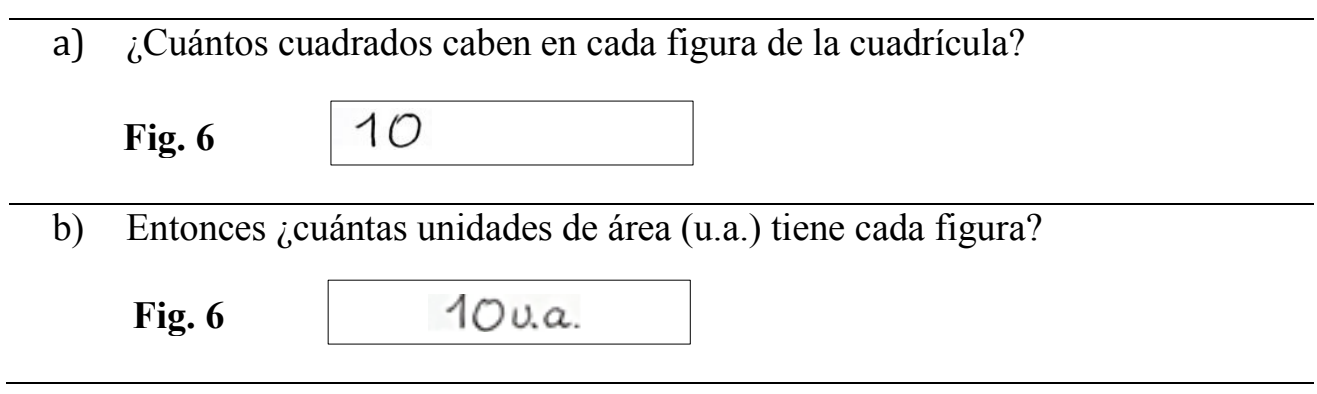

Fuente: Adaptado de Borja (2015, p. 49)

De esta manera, la estudiante Melissa contesta en la ficha de la actividad 1 con respecto al trapecio rectángulo, identificado con Fig. 6. En la cual, con respecto al ítem a) menciona solo la cantidad.

Asimismo, en el análisis a posteriori la estudiante Viviana a diferencia de lo previsto en el a priori, en la modificación mereológica del trapecio rectángulo obtiene las siguientes subfiguras: un triángulo, un pentágono y un hexágono según los trazos hechos a lápiz por la estudiante en la Fig. 6. Luego, realiza la modificación posicional al trasladar dos de estas subfiguras que son el triángulo y el pentágono hacia el exterior de la figura inicial, según indica las flechas que trazó. Para formar un hexágono cóncavo, al reagrupar estas sub-figuras, es decir, realiza la operación de reconfiguración según se aprecia con los trazos hechos a lápiz por la estudiante, obteniéndose una figura geométrica que no se esperaba. Pensamos que ella 
obtuvo esta figura al tener como prioridad completar los cuadrados que faltaban en el trapecio rectángulo para obtener la medida del área y además su aprehensión perceptiva de ver las subfiguras al trasladarse encajen al completar los cuadrados (ver Figura 5).

Figura 5: Descomposición heterogénea del trapecio rectángulo

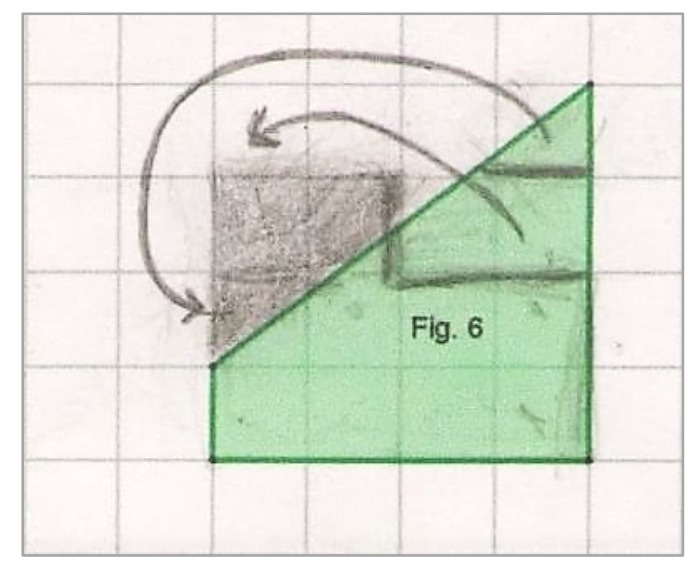

Fuente: Adaptado de Borja (2015, p. 57)

Borja (2015), indica que la estudiante Viviana responde a los ítems a) y b) de la actividad 1: Trabajemos con la malla cuadriculada, al realizar la estrategia del conteo, según lo previsto en el a priori (ver Tabla 2).

Tabla 2: Respuestas de Melissa con respecto al trapecio isósceles de la actividad 1

a) ¿Cuántos cuadrados caben en cada figura de la cuadrícula?

Fig. 6

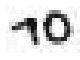

b) Entonces ¿cuántas unidades de área (u.a.) tiene cada figura?

Fig. 6

$$
10 \mathrm{U}, \mathrm{a}
$$

Fuente: Adaptado de Borja (2015, p. 58)

De esta manera, la estudiante Viviana contesta en la ficha de la actividad 1: Trabajemos con la malla cuadriculada con respecto al trapecio rectángulo, identificado con Fig. 6. En la cual, con respecto al ítem a) también menciona solo la cantidad.

En el análisis a posteriori del estudiante Alcides a diferencia de lo previsto en el a priori, en la modificación mereológica que realiza en el trapecio rectángulo obtiene dos sub-figuras que son las siguientes: un trapecio rectángulo y un hexágono, según indica el trazo realizado en la Fig. 6. Luego, en la modificación posicional trasladó el trapecio rectángulo obtenido hacia el exterior de la figura inicial para obtener un hexágono cóncavo, al reconfigurar, es decir, reagrupar estas dos sub-figuras. De esta manera, completa los cuadrados considerados como 
unidades de área. Pensamos que su aprehensión perceptiva del estudiante le permite ver las figuras geométricas que encajen para completar los cuadrados sin realizar muchos trazos. (Ver figura 6).

Figura 6: Descomposición heterogénea del trapecio rectángulo

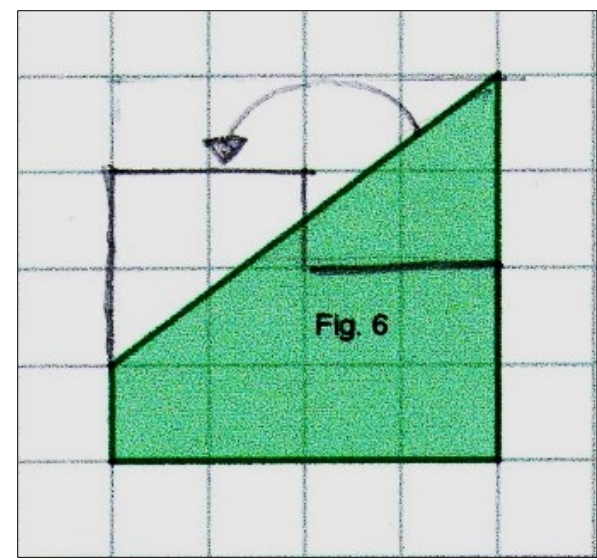

El estudiante Alcides responde a los ítems a) y b) de la actividad 1: Trabajemos con la malla cuadriculada, al realizar la estrategia del conteo según lo previsto en el a priori (ver Tabla 3).

Tabla 3: Respuestas de Melissa con respecto al trapecio isósceles de la actividad 1

a) ¿Cuántos cuadrados caben en cada figura de la cuadrícula?

Fig. 6

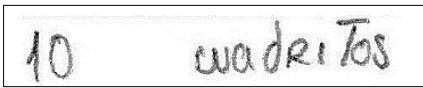

b) Entonces ¿cuántas unidades de área (u.a.) tiene cada figura?

Fig. 6

$10 \quad 4 . a$

Fuente: Adaptado de Borja (2015, p. 49)

Además, el estudiante Alcides contesta en la ficha de la actividad 1: Trabajemos con la malla cuadriculada con respecto al trapecio rectángulo, identificado con Fig. 6. En la cual, podemos apreciar que la respuesta que da en el ítem a "10 cuadritos" no la esperábamos. Pensamos que menciona la palabra cuadritos por la presencia de la cuadrícula.

\section{Conclusiones}

Podemos mencionar que los estudiantes, del segundo grado de Educación Secundaria de la Institución Educativa “Andahuasi” de gestión pública, al realizar la modificación mereológica en el trapecio rectángulo identificado con Fig. 6 obtuvieron cantidades de sub-figuras diferentes a lo previsto en el análisis a priori. Melissa obtuvo más sub-figuras (cinco) ya que 
pensamos que se apoyó en la cuadrícula que presentaba la malla por sus trazos realizados. Mientras, Viviana y Alcides obtuvieron menos sub-figuras (tres y dos, respectivamente) de lo previsto, pensamos que en ellos la cuadrícula no fue tan determinante para obtener dichas subfiguras, ya que su aprehensión perceptiva les permitió ver más allá de la cuadrícula figuras geométricas que al ser trasladadas completen los cuadrados del trapecio rectángulo que estaban incompletos para obtener la medida de área de la figura inicial. Asimismo, estas subfiguras obtenidas son por descomposición heterogénea porque son de forma diferentes entre sí.

Luego, realizan la modificación posicional al trasladar las sub-figuras que creen conveniente para completar los cuadrados de la cuadrícula, según indican las flechas trazadas con el lápiz por cada uno de los estudiantes. Para realizar la operación de reconfiguración al reagrupar dichas sub-figuras geométricas y formar una nueva figura diferente a la figura inicial que permita realizar la estrategia del conteo para determinar la medida de área. De tal manera, Melissa obtiene un rectángulo como se esperaba al realizar la reconfiguración. Sin embargo, Viviana y Alcides obtienen un hexágono cóncavo. Pero, en los tres casos logran obtener la medida de área que es 10 u.a. al emplear la estrategia del conteo de los cuadrados completos, considerados como unidades de área. halló la medida del área del trapecio isósceles. Los estudiantes entonces realizaron tratamientos en el registro figural como fue la operación de reconfiguración.

Por otro lado, al contestar los estudiantes la pregunta a) ¿Cuántos cuadrados caben en cada figura de la cuadrícula? Melissa y Viviana menciona solo una cantidad numérica que es 10 , mientras que Alcides menciona 10 cuadritos. Al respecto, pensamos que les cuesta llamarlos como figuras geométricas que son cuadrados. En tanto, que para la pregunta b) Entonces, ¿cuántas unidades de área (u.a.) tiene cada figura?, los estudiantes respondieron como se esperaba 10 u.a., podemos expresar que reconocen que cada cuadrado de la malla cuadriculada es una unidad de área.

Borja (2015) menciona que no solo se puede hallar la medida del área del trapecio isósceles con uso de la fórmula del área, "sino también con realizar tratamientos en la figura como la operación de reconfiguración" (p. 76).

\section{Agradecimientos}

Debemos manifestar nuestro agradecimiento en el desarrollo de esta investigación a las siguientes instituciones: Línea de investigación “Tecnologías y Visualización en Educación 
Matemática" de la Maestría en Enseñanza de las Matemáticas de la Pontificia Universidad

Católica del Perú-PUCP; al grupo de investigación Tecnologías y Visualización en Educación Matemática del Instituto de Investigación sobre Enseñanza de las Matemática a la Institución Educativa Básica Regular “Andahuasi” y al proyecto internacional Processos de Ensino e Aprendizagem de Matemática em Ambientes Tecnológicos PEA-MAT/DIMAT” proceso 2013/23228-7 e, IREM-PUCP - PI0272.

\section{Referencias}

Alva, F. (2015). Geometría. Teoría y práctica. Lima Perú: Editorial san Marcos.

Artigue, M. (1995). Ingeniería didáctica en educación Matemática. Un esquema para la investigación y la innovación en la enseñanza y aprendizaje del cálculo. Bogotá, Colombia. Editorial Iberoamérica.

Borja, I. (2015). Reconfiguración del trapecio para determinar la medida del área de dicho objeto matemático con estudiantes del segundo grado de educación secundaria. (Tesis de Maestría en Enseñanza de las matemáticas). Pontificia Universidad Católica del Perú. Lima, Perú.

Douady, R. \& Perrin-Glorian, M. (1987). Um processus d'apprentissage du concept d'aire de surface plane. Cahier de didactique des mathématiques-IREM. (37). Université Paris VII. Recuperado de http://www.irem.univ-paris-diderot.fr/up/publications/IPS00015.pdf

Duval, R. (1988). Approche cognitive des problèmes de géométrie en termes de congruence. IREM 1, pp. 57-74. Recuperado de https://mathinfo.unistra.fr/fileadmin/upload/IREM/Publications/Annales_didactique/vol_01/ads c1_1988-004.pdf

Duval, R. (1994). Les différents fonctionnements d'une figure dans une demarche géométrique. Repères-IREM, (17), pp. 121-138. Recuperado de http://www.univirem.fr/exemple/reperes/articles/17_article_119.pdf

Duval, R. (2001). Los problemas fundamentales en el aprendizaje de las matemáticas y las formas superiores del desarrollo cognitivo. (Myriam Vega, trad.). Santiago de Cali, Colombia: Universidad del Valle, Instituto de Educación y Pedagogía, Grupo de Educación Matemática. (Obra original publicada en 1999).

Duval, R. (2004). Semiosis y pensamiento humano. (Myriam vega, trad). Cali, Colombia: Universidad del valle, Instituto de Educación y Pedagogía, Grupo de Rducación Matemática. (Obra original publicada en 1999).

Duval, R. (2005). Les conditions cognitives de l'apprentissage de la géométrie: développement de la visualisation, différenciation des raisonnements et coordination de leurs fonctionnements. Repères-IREM 10, pp. 5-53. Recuperado de https://mathinfo.unistra.fr/fileadmin/upload/IREM/Publications/Annales_didactique/vol_10/ads c10-2005_001.pdf

Perú, Ministerio de Educación (2012). Matemática 2 Secundaria. Lima, Perú: Editorial Norma. 